\title{
Color Doppler Ultrasound and MRI Findings of Vein of Galen Malformation in a Newborn, a Case Report.
}

\author{
Jiraporn Srinakarin ${ }^{1}$, Jureerat Thammaroj ${ }^{1}$, Ratana Kumwilaisak ${ }^{2}$ \\ Waranon Munkong ${ }^{1}$, Junya Jirapradittha ${ }^{3}$ \\ ${ }^{1}$ Department of Radiology, Faculty of Medicine, Khon Kaen University, Thailand. \\ ${ }^{2}$ Department of Obstetrics and Gynecology, Faculty of Medicine, Khon Kaen University, Thailand. \\ ${ }^{3}$ Department of Pediatrics, Faculty of Medicine, Khon Kaen University, Thailand.
}

\begin{abstract}
The Vein of Galen aneurysmal malformation (VGAM) is a rare intracranial arteriovenous anomaly that has usually been diagnosed prenatally. We reported a near term boy, 2,140 grams body weight, with a large VGAM, who was diagnosed prenatally by color Doppler ultrasound. After birth, his APGAR scores were 2, 4 , and 8 , respectively. An emergency cranial ultrasound was performed promptly when his vital sign began to be stable. The image revealed a huge dilatation of the great vein of Galen, measured about $1.9 \times 2.0 \times$ $3.8 \mathrm{~cm}$. in diameter, with mixed venous and arterial flow profiles. Abnormal dilatation of the right internal carotid artery, and circle of Willis were also identified and likely to be an arterial feeder to choroidal artery which directly draining into the great vein of Galen. MRI, MRA, and MRV of the brain were performed on the following day and also showed a huge aneurysmal dilatation of a median vein of prosencephalon (precursor of the great vein of Galen) and marked dilatation of falcine sinus. Torcular herophili, both transverse / sigmoid sinuses and both internal jugular veins showed abnormal dilatation on MRA and MRV. Endovascular transarterial embolization was planned to performe on this patient but his vital sign was not stable. He finally expired from severe congestive heart failure after 5 days of life.
\end{abstract}

Keywords: Color Doppler ultrasound, MRI, VGAM 


\section{Introduction}

The Vein of Galen aneurysmal malformation (VGAM) is a rare congenital disease with an incidence of 1:25000. It was first described by Steinhel et al in $1895^{1}$. The VGAM is located in the midline in the choroidal fissure. It consists of multiple feeding arteries including anterior and posterior choroidal arteries, anterior cerebral artery. The feeding arteries drain directly into a large venous pouch called the vein of Galen. Raybaud et al reported that malformation develops between the $6^{\text {th }}$ and $11^{\text {th }}$ weeks of gestation and thought to result from the development of an arteriovenous connection between primitive choroidal vessels and the median prosencephalic vein of Markowski ${ }^{2}$. Base on angioarchitecture association, the VGAM are divided into choroidal and mural types. The Vein of Galen aneurysmal malformation (VGAM) can be diagnosed by many imaging modalities such as color Doppler sonography, CT scan and MRI. It is usually or accidentally been diagnosed prenatally ${ }^{3,4}$. Identification of the type of the VGAM by the imaging helps us to know clinical outcome and prognosis of the neonate. It is also very important to distinguish the VGAM from aneurysmal dilatation of the true vein of Galen caused by an adjacent brain AVM called VGAD (vein of Galen aneurysmal dilatation) which has a higher risk of hemorrhage ${ }^{5}$.

We reported a 37 weeks gestation age boy with 2,140 grams body weight, who was diagnosed VGAM prenatally by color Doppler ultrasound. The boy was delivered by Cesarean section with APGAR scores of 2, 4, and 8, respectively. Prenatal diagnosis of a large $(5 \mathrm{~mm})$ patent ductus arteriosus (PDA) was reported with mild dilated coronary sinus and cardiac chambers. Tricuspid regurgitation was also identified with pressure gradient of $37 \mathrm{~mm} \mathrm{Hg}$ and
EF 32\%. An emergency transcranial Doppler sonography was performed in the NICU when his vital sign began to be stable. The color Doppler ultrasound (LOGIQ P6, GE Medical System, Milwaukee, WI, USA) revealed a huge dilatation of the great vein of Galen, measured about $1.9 \times 2.0 \times 3.8 \mathrm{~cm}$. in diameter, with mixed venous and arterial flow profiles (Fig.1). Multiple high velocity (>100 cm/s) and dilated feeding vessels were clearly identified draining directly into the great vein of Galen (Fig.2). MRI, MRA, and MRV of the brain (Philips Achieva 3.0T TX, Netherlands) were performed on the following day and also showed a huge aneurysmal dilatation of a median vein of prosencephalon (precursor of the great vein of Galen) and marked dilatation of falcine sinus (Fig.3). Torcular herophili, both transverse/sigmoid sinuses and both internal jugular veins also showed abnormal dilatation on MRA and MRV. The diagnosis of VGAM was reported with likely be a choriodal type. We planned to perform endovascular transarterial embolization when his clinical and vital sign were stable. His parents were referred for consultation with a pediatric specialist, a cardiovascular surgeon, a neurointerventionist and a palliative team in order to plan an optimal management and let them know the prognosis. Clinically, his heart failure was progressing and superimposed with anemia and septicemia. Finally, he expired on the $5^{\text {th }}$ day of life from cardiovascular failure.

\section{Discussion}

The vein of Galen malformation is actually a misnomer term because the ectatic vein we identified was the median mesencephalic vein of Markowski, the embryonic precursor of the vein of Galen and is normally absent in the adult ${ }^{2}$. It's divided into two types; the choroidal and mural types. The former 


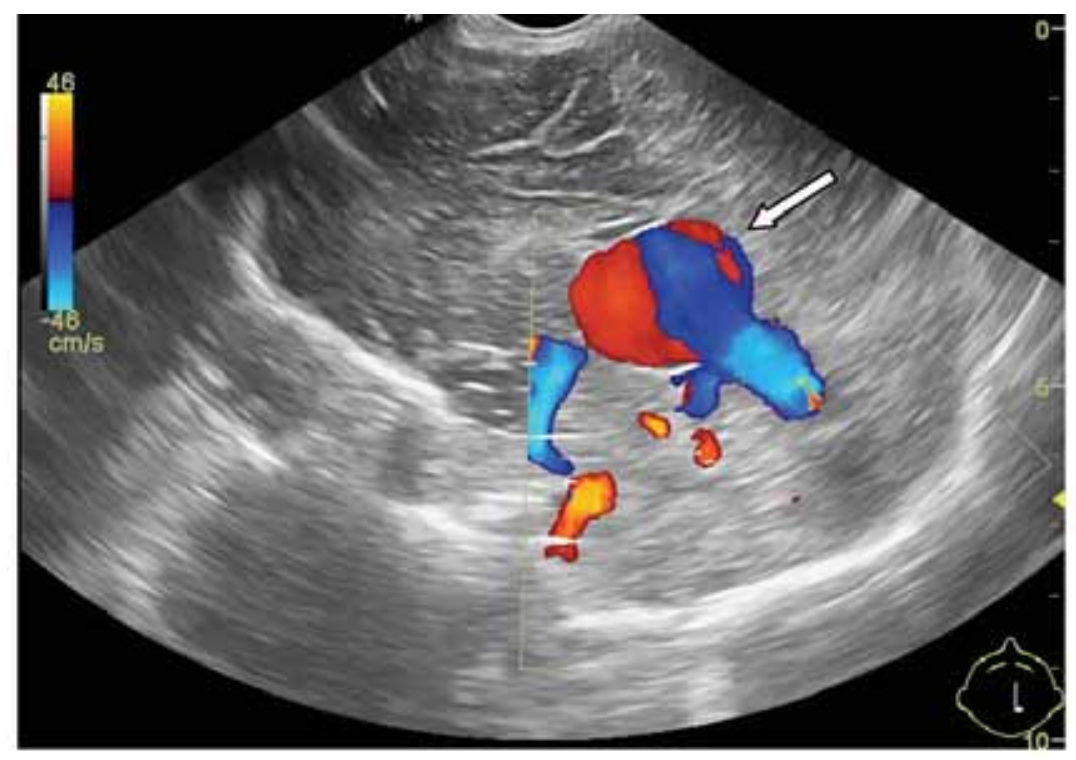

Fig 1. Transcranial color Doppler sonogram shows turbulent flow (Yin-Yang sign) in the aneurysmal dilatation of the great vein of Galen (arrow).

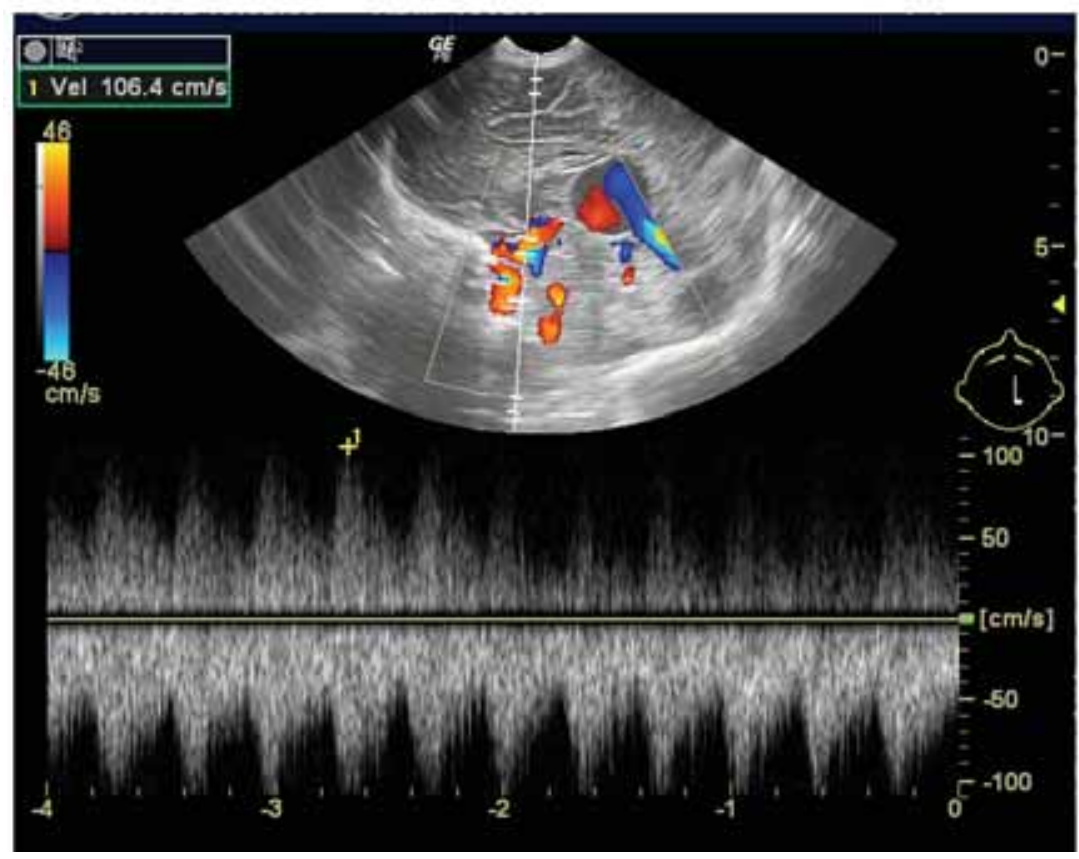

Fig 2. Transcranial color Doppler sonogram shows abnormal high velocity of the spectral waveform of the feeding artery (arrow). 


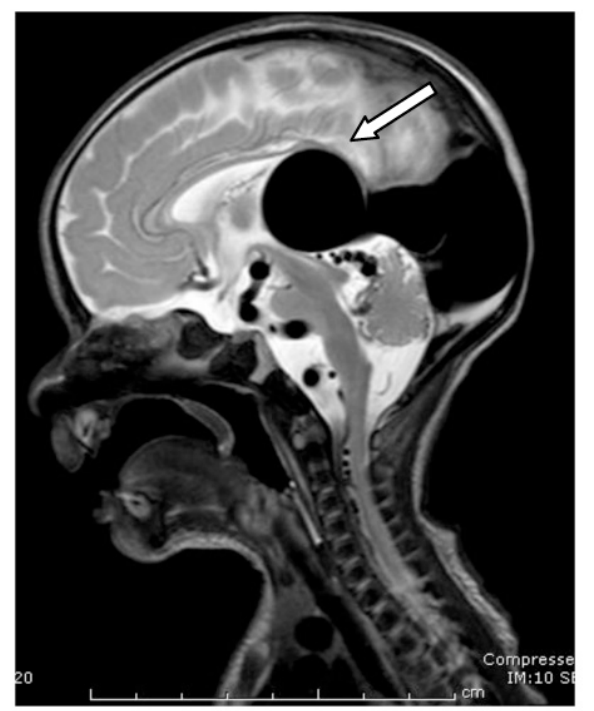

Fig 3. A sagittal T2-weighted MRI of the brain shows a markedly enlarged median prosencephalic vein of Markowski, or the great vein of Galen (arrow) with marked dilatation of the straight sinus and the Torcular herophili.

type receives feeding arteries from choroidal arteries before emptying into a venous pouch, and is a primitive condition associated with poor clinical outcome in neonates $^{6-9}$. The latter type is characterized by direct AVFs within the wall of the median prosencephalic vein of Marlowski and is found in infants with better prognosis. We reported a choroidal type of VGAM in the neonate associated with severe high output heart failure. Transcranial sonography is the best imaging modality for definite diagnosis as well as during late prenatal ultrasound scanning ${ }^{10}$. MRI with MR angiography helps us identifying the arterivenous malformations and a huge great vein of Galen (in fact it is the median prosencephalic vein of Markowski). MRA is also useful as a preliminary noninvasive imaging plan, and a follow up before performing endovascular transarterial embolization. The therapy of choice is the endovascular embolization using a special glue ${ }^{6-9}$. The therapeutic efficacy of the treatment reported by Lasjaunias et al, using the 21-point scale in neonate based on a number of factors, including cardiac, cerebral, hepatic, respiratory, and renal function those help a neurointerventionist and a pediatrician to predict the proper time to perform endovascular embolization for a good outcome ${ }^{9}$. In a large scale study by Lasjaunias et al reported a mortality rate of $10.6 \%$ overall, and $52 \%$ for neonate ${ }^{9}$. In our case report, he expired from severe high output heart failure, regarded 21-point scale by Lasjaunias. His scale was less than 8 suggested that endovascular therapy would be unsuccessful and thus treatment was not indicated.

\section{Acknowledgment}

We thank the Departments of Pediatrics, Obstetrics and Gynecology, and Radiology at the Faculty of Medicine and the Medical Records Divisions at Srinagarind Hospital, Khon Kaen University, for their supportive cooperation and Professor Tula Dhiensiri for assistance with the English-language presentation of the manuscript. 
January-April 2013, Volume XIX No.I

\section{References}

1. Dandy W.E., "Experimental hydrocephalus," Annals Surgery, 1919;70(2)129-42.

2. Raybaud CASC, Strother CM, Hald JK. Aneurysms of the vein of Galen: embryonic considerations and anatomical features relating to the pathogenesis of the malformation. Neuroradiology 1989;31:109-28.

3. Beucher G, Fossey C, Belloy F. Richter B, Dreyfus M. Antenatal diagnosis and management of vein of Galen aneurysm: review illustrated by a case report. Journal de Gynecologie Obstetrique et Biologie de la Reproduction;2005:34(6);613-9.

4. Yamashita Y, Abe T, Ohara N, et al. Successful treatment of neonatal aneurymal dilation of the vein of Galen: the role of prenatal diagnosis and trans-arterial embolization. Neuroradiology1992;34(5):457-9.

5. Bhattacharya J J, Thammaroj J. Vein of Galen Malformations. J Neurol Neurosurg Psychiatry 2003;74(Suppl I):i42i44.
6. Lasjaunias PL, Ter Brugge K, Lopez Ibor L, et al. The role of dural anomalies in vein of Galen aneurysm: report of six cases and review of literature. AJNR Am J Neuroradiol 1987;8:185-92.

7. Lasjaunias PL, Garcia-Manaco R, Rodesch G, Ter Brugge K, Zerah M, Tardieu M, et.al. Vein of Galen Malformation. Endovascular management of 43 cases. Childs Nerv Syst 1991;7:360-7.

8. Lasjaunias PL, Hui F, Zerah M, Garcia-Manaco R, Malherbe V, Rodesch G, et al. Cerebral arteriovenous malformations in children. Management of 179 consecutive cases and review of the literature. Childs Nerv Syst1995;11:6679 .

9. Lasjaunias PL, Chng SM, Sachet M, Alvarez H, Rodesch G, Garcia-Manaco R. The management of vein of Galen aneurysmal malformations. Neurosurgery 59 (5 Suppl 3); 2006:S3-113,S184-94.

10. Stephen S, Rodesch G, Elolf E, Wiemann D, Jorch G. Vein of Galen aneurysmal malformations: an ultrasonographic incidental finding-a case report. Pediatrics 2012; doi:10.1155/2012/824284(6pages). 